\title{
Procesos de adaptación social de estudiantes adolescentes migrantes venezolanos a través de la inclusión académica al sistema educativo colombiano en básica secundaria
}

\author{
Social adaptation processes of Venezuelan migrant adolescent students through \\ academic inclusion in the Colombian education system in basic secondary \\ Processos de adaptação social de adolescentes migrantes venezuelanos através \\ da inclusão acadêmica no sistema colombiano de ensino médio
}

\author{
JESÚS DAVID RUBIO ORTEGA ${ }^{1}$ \\ ELOY MAYA PÉREZ ${ }^{2}$
}

\begin{abstract}
RESUMEN
El estudio surge de la intervención realizada con alumnos adolescentes venezolanos migrados a la Garita norte de Santander, Colombia. El propósito del proyecto se centró en describir procesos de adaptación al territorio colombiano a través de la inclusión al sistema educativo de ese país de estudiantes migrantes venezolanos. Las razones de la movilidad responden a diversos factores y causan diversos efectos en la población que se moviliza como en el territorio que los recibe. Entre estos efectos se destacan aquellos que tienen que ver el logro de la adaptación al nuevo contexto. Se concluye que para los participantes la migración fue favorable en términos personales ya que el apoyarse en la escuela repercutió en su sentido de pertenencia y autorrealización.
\end{abstract}

Palabras clave: Migración; adolescentes; adaptación académica; sistema educativo colombiano.

\begin{abstract}
The study arises from the intervention carried out with Venezuelan adolescent students migrated to the Garita north of Santander in Colombia. The purpose of the project focused on describing processes of adaptation to the Colombian territory through the inclusion of Venezuelan migrant students in the educational system of that country. The reasons for mobility respond to various factors and cause various effects on the population that is mobilized as well as on the territory that receives them. These effects include those that have to do with achieving adaptation to the new context. It is concluded that for the participants, migration was favorable in personal terms, since leaning at school had an impact on their sense of belonging and self-realization.
\end{abstract}

Keywords: Migration; teenagers; academic adaptation; Colombian educational system. 


\section{RESUMO}

O estudo decorre da intervenção realizada com alunos adolescentes venezuelanos migrados para a Garita norte de Santander, Colômbia. O objetivo do projeto era descrever os processos de adaptação ao território colombiano, através da inclusão de estudantes migrantes venezuelanos no sistema educacional daquele país. As razões da mobilidade respondem a vários fatores e causam vários efeitos na população mobilizada e no território que a recebe. Esses efeitos incluem aqueles que têm a ver com a adaptação ao novo contexto. Em conclusão para os participantes, a migração foi favorável em termos pessoais, uma vez que a inclinação na escola teve impacto no senso de pertencimento e autorrealização.

Palavras-chave: Migração; adolescentes; adaptação acadêmica; Sistema educacional colombiano.

\section{INTRODUCCIÓN}

La migración es un fenómeno constante producto de factores estructurales propios de cada región. La OPS afirma que los conflictos sociales y políticos, la inseguridad alimentaria, los desastres naturales, el cambio climático, la degradación ambiental, los problemas económicos, la violencia forman parte de tales factores adversos y problemas estructurales que han impedido que las personas construyan medios de subsistencia saludables y sostenibles, y les ha obligado a buscar mejores condiciones de vida en otros sitios para ellas y sus familias.

En cuanto a lo referente a las migraciones, éstas están catalogadas por varios autores como un acontecimiento que se ha ido incrementando en los tiempos recientes, debido entre otras causas a las desigualdades entre las poblaciones, también a fenómenos como el de globalización, los avances en los medios de comunicación, la tecnología, el transporte, entre otros. Dando como resultado una gran masa de personas que se trasladan con pocos bienes y recursos a zonas más privilegiadas con la finalidad de obtener mejor calidad de vida. Con respecto en lo anterior, los acontecimientos migratorios se suceden por la movilidad de una persona o un grupo de éstas, que buscan nuevas opciones de subsistencia, ya sea para trabajar o realizar estudios en la población residente, de acuerdo con la finalidad por la que migre el individuo puede convertirse en un inmigrante temporal o permanente produciéndose primero una salida de un país con una posterior llegada a una región la cual va a habitar y es diferente a la zona donde nació.

Por consiguiente, para Puyol (1990, citado por López, 2015), las migraciones consisten en un "conjunto de movimientos que tienen por objetivo trasladar la residencia de las personas interesadas de un lugar de origen a otro de destino. Una migración supone siempre el desplazamiento de un grupo importante de personas". De acuerdo con el autor, las migraciones tienen que ver con el traslado de personas de una zona a otra con diferentes objetivos, la misma debe poseer como característica el flujo de una gran cantidad de individuos a la región de destino provocando cambios significativos en la zona donde llegan los inmigrantes. En este sentido, Kearney \& Bernadete (2002, p. 38) definieron la migración como "un movimiento que atraviesa una frontera significativa que es definida y mantenida por cierto régimen político: un orden formal o informal de tal manera que afecta la identidad del individuo". 
Entonces, la migración como fenómeno social según lo pautado anteriormente consiste en el paso entre zonas fronterizas que poseen ciertas leyes y normativas, las cuales los países implicados deben hacer cumplir, a su vez estos reglamentos le proporcionan legitimidad a los cuales los individuos deben someterse, perturbando positiva o negativamente la forma de ser de la persona. Pero al mismo tiempo la migración se ha vuelto un interesante tema de estudio de los investigadores en ciencias sociales que buscan explicar no solo las dimensiones económico-políticas sino dar visibilidad a los procesos humanos implicados llevando incluso las discusiones a la reflexión sobre la calidad y condición de las personas involucradas, La Fundación García (2002), llama migrantes exclusivamente a aquellos extranjeros originarios de países económicamente más desfavorecidos y para los procedentes de países desarrollados el término extranjero.

\section{La migración y sus implicaciones en los procesos educativos en la frontera Colombia-Venezuela}

En octubre de 2018 el informe preparado para el Banco Mundial sobre la crisis de la frontera colombo-venezolana y que se tituló Migración desde Venezuela a Colombia: impactos y estrategia de respuesta en el corto y mediano plazo $^{3}$ lo adelantaba haciendo una especie de radiografía cuando dice:

Los migrantes se caracterizan por tener condiciones socioeconómicas complejas. Esto es particularmente cierto para los migrantes irregulares. La población migrante tiene dos veces mayor probabilidad de estar desempleada que la población de las áreas receptoras y cerca de la mitad viven en condiciones de pobreza. En términos de salud, la población venezolana se enfrenta a una creciente desprotección en la atención médica, particularmente afectando a los migrantes irregulares, quienes están casi en su totalidad fuera del sistema de salud. Igualmente, la migración afecta el acceso y permanencia en el sistema escolar de niños y jóvenes, con un nivel de inasistencia escolar de cerca del 40\% entre todos los migrantes, más del doble que la tasa de la población en áreas receptoras. La inasistencia escolar entre los migrantes irregulares es casi el doble que la de toda la población migrante.

Los sistemas educativos no fueron pensados para las personas en desplazamiento que supone la migración masiva (López, 2008) que se produce desde los países económicamente menos desarrollados los más prometedores en términos de seguridad laboral que garanticen la cierta calidad de vida. Diversas investigaciones se han enfocado en analizar los procesos de desplazamiento y asentamiento de migrantes forzados; estudios como el de Lasso (2013) \& Vega (2013) se han enfocado en identificar las circunstancias que afectan a la población en situación de desplazamiento cuando ingresan al sistema escolar, Hernández (2009), afirma que entre estas circunstancias destacan el hecho de que los migrantes se constituyen como sujetos de derecho y sujetos colectivos que son reivindicados a través de la educación. Duque \& Lasso (2016) señalan que en el caso colombiano -desde 1995- se inicia la regulación de marcos normativos y orientaciones para la atención de población en situación de desplazamiento; sin embargo, aún no han sido de cobertura suficiente para impactar lo que los docentes requieren conocer y hacer al respecto. Es pertinente mostrar cómo los estudiantes van respaldando esta teoría con sus testimonios y de esta manera ayudan a ilu-

3 Este documento se puede consultar en https://r4v.info/es/documents/download/66643 
minar si las causas o motivaciones de su salida del país de origen se ajusta a la realidad o es puro invento como afirman algunos.

ACNUR (2012) publicaba un informe en el que se reconocía una cifra aproximada de unos 3,4 millones de venezolanos que salían de su país de manera regular, esta cifra desde luego a medida que pasa el tiempo va variando porque la salida de venezolanos sigue en aumento, para efectos del presente informe es solo un punto de referencia. De esos miles de venezolanos algunos han llegado a instalarse a la Frontera, específicamente se han ubicado en los municipios de Villa del Rosario, de los Patios y de la Ciudad de Cúcuta entre otros. En este mismo sentido en octubre de 2018 el informe preparado para el Banco Mundial, en Migración desde Venezuela a Colombia: impactos y estrategia de respuesta en el corto y mediano plazo, lo adelantaba haciendo una especie de radiografía cuando dice:

Los migrantes se caracterizan por tener condiciones socioeconómicas complejas. Esto es particularmente cierto para los migrantes irregulares. La población migrante tiene dos veces mayor probabilidad de estar desempleada que la población de las áreas receptoras y cerca de la mitad viven en condiciones de pobreza. En términos de salud, la población venezolana se enfrenta a una creciente desprotección en la atención médica, particularmente afectando a los migrantes irregulares, quienes están casi en su totalidad fuera del sistema de salud. Igualmente, la migración afecta el acceso y permanencia en el sistema escolar de niños y jóvenes, con un nivel de inasistencia escolar de cerca del $40 \%$ entre todos los migrantes, más del doble que la tasa de la población en áreas receptoras. La inasistencia escolar entre los migrantes irregulares es casi el doble que la de toda la población migrante.

En relación con lo anteriormente expuesto, se propuso como objetivo describir procesos de adaptación académica al sistema educativo colombiano de estudiantes migrantes venezolanos en básica secundaria en la institución educativa la Garita de los Patios norte de Santander- Colombia a partir de la narración de la propia experiencia de los alumnos migrados. Por lo que, la presente investigación está sustentada en la siguiente pregunta ¿Cómo son los procesos de adaptación académica al sistema educativo colombiano de estudiantes migrantes venezolanos en básica secundarias en la institución educativa la Garita de los Patios norte de Santander- Colombia?

\section{METODOLOGÍA}

Es un diseño cualitativo de corte etnográfico que se desarrolló durante 2019. Partiendo de la idea que comparte Patton (2014) sobre los diseños etnográficos mediante los que, entre otras cosas, se pretenden describir y analizar ideas, creencias, significados, conocimientos y prácticas de grupos, culturas y comunidades. Creswell (2005) señala que los diseños etnográficos estudian categorías, temas y patrones referidos a las culturas.

Esta experiencia se fundamentó en un diseño etnográfico, en el cual se recolectaron datos a través de la acción directa -observación participante- con los alumnos venezolanos migrados a la Garita norte de Santander, Colombia, a través de la guía de entrevista que se diseñó ex professo para este proyecto. Se trabajó con los alumnos migrados a los cuales se les aplicó la entrevista, de estas se retoman algunos diálogos que se exponen en el apartado siguiente mediante los cuales se da cuenta tanto del proceso de adaptación en este contexto 
migratorio a través de la inclusión en este nuevo territorio. Ahora bien, aunque se partió de la observación participante como ejercicio investigativo, el estudio se fortaleció con la ayuda de la etnografía que facilitó detallar las realidades de los participantes en relación con su experiencia migratoria con la intención de desarrollar una comprensión de las mismas y de la diáspora.

Teniendo en cuenta lo expuesto por Ramírez (1994) acerca de que la observación participante implica una interacción, investigador-sujetos investigados. Se decide dar paso a mediados del año 2018 de una observación no participante a la observación participante y es que de hecho ya se interactuaba con ellos en clases impartidas diariamente, en el desarrollo de los proyectos pedagógicos de aprendizaje y de transversalidad, en actividades de formación cultural, cívica y religiosa, lo que permite tener un acercamiento a los estudiantes conociendo de manera directa sus maneras de pensar y de actuar, sus emociones, ideales, frustraciones y limitaciones es decir que con su quehacer diario dan cuenta de su experiencia en el nuevo país. Asimismo, Ramírez (1994) sostiene que los sujetos pueden aportar comentarios sobre hechos y sucesos, anécdotas, opiniones y conceptos y, al mismo tiempo, el investigador puede observar una serie de actividades, conductas y manifestaciones de diversa índole y los sujetos investigados proporcionar interpretaciones respecto a sus acciones y a la forma como perciben los resultados que obtienen.

\section{RESULTADOS}

Debido al rigor etnográfico de la investigación, en el análisis, se llevó a cabo un proceso hermenéutico, con énfasis en la producción de categorías, para caracterizar las evidencias presentes en la realidad. Para tal efecto, se diseñó una guía de entrevista semiestructurada enfocadas en la propia experiencia de los alumnos migrados; con la información obtenida se articularon dos categorías de análisis que, para el caso de esta intervención socioeducativa, describen los procesos de adaptación académica al sistema educativo colombiano de los estudiantes migrantes venezolanos.

Estas categorías revisan dos temas: a) razones de la movilidad y b) experiencias de adaptación al contexto colombiano a través de la educación. Esta dualidad permitió establecer un orden lógico entre los significados de la migración y los mecanismos que usarán para adaptarse. El análisis se describe a continuación en forma narrativa y se comparten algunos fragmentos de las entrevistas para dar sentido al análisis de las categorías.

\section{Razones para la movilidad}

De entre las preguntas elaboradas en la guía de entrevista se seleccionaron dos con las que se orientó el análisis de la información ¿Por qué tú y tu familia se vinieron de Venezuela? ¿Qué necesidades los llevo a dejar su patria? A través de la narrativa de los participantes, fue posible identificar cuatro causas a saber que se explican a través de algunos diálogos. 
Tabla 1.

Causas de la migración desde la perspectiva de los estudiantes

\begin{tabular}{|c|c|c|c|}
\hline $\begin{array}{c}\text { Crisis socio } \\
\text { política }\end{array}$ & $\begin{array}{c}\text { Crisis } \\
\text { económica }\end{array}$ & $\begin{array}{c}\text { Dificultades en el } \\
\text { servicio de salud }\end{array}$ & $\begin{array}{c}\text { Búsqueda de estabilidad en el } \\
\text { servicio educativo }\end{array}$ \\
\hline Pobreza & Pobreza & $\begin{array}{c}\text { Dificultad para el acceso } \\
\text { a la salud }\end{array}$ & $\begin{array}{c}\text { Búsqueda de nuevas oportunida- } \\
\text { des educativas }\end{array}$ \\
\hline Necesidad & Necesidad & $\begin{array}{c}\text { Alto costo para la aten- } \\
\text { ción de la enfermedad }\end{array}$ & Acceso a los servicios educativos \\
\hline $\begin{array}{c}\text { Crecimiento de la } \\
\text { inflación }\end{array}$ & $\begin{array}{c}\text { Crecimiento de } \\
\text { la inflación }\end{array}$ & Escases de medicinas & $\begin{array}{c}\text { Educación libre para la movilidad } \\
\text { y el desarrollo personal y social }\end{array}$ \\
\hline $\begin{array}{c}\text { Búsqueda de nue- } \\
\text { vas oportunidades } \\
\text { laborales }\end{array}$ & $\begin{array}{c}\text { núsqueda de } \\
\text { nuevas oportu- } \\
\text { nidades labo- } \\
\text { rales }\end{array}$ & $\begin{array}{c}\text { Servicios de salud defi- } \\
\text { cientes o sin cobertura }\end{array}$ & \\
\hline & & & \\
\hline
\end{tabular}

A quienes llegaron a vivir en el Municipio de los Patios y de manera concreta llegaron a estudiar en la institución educativa se les preguntó sobre las causas que les obligo a salir de su país, con el fin de saber cuáles son las motivaciones reales y con la intención de despejar cualquier tipo de duda creada por algún sesgo político o mediático.

Es pertinente mostrar como los estudiantes van respaldado esta teoría con sus testimonios y de esta manera ayudan a iluminar si las causas o motivaciones de su salida del país de origen se ajusta a la realidad o es puro invento como afirman algunos.

\section{Experiencias de adaptación al contexto colombiano}

Después de haber hecho un análisis sobre las causas de su salida y su llegada a Colombia, se indagó sobre la transición que experimentan los jóvenes que va más allá de un cambio de lugar; esto es, la entrevista se dirigió a detectar, entre otros factores, procesos de adaptación al territorio colombiano a través de la inclusión al sistema educativo de estudiantes migrantes venezolanos. De la guía de preguntas que se elaboró para realizar este proyecto, se retomaron las siguientes para realizar el análisis de esta categoría:

- ¿Qué es lo más duro para ti de este proceso de adaptación a la vida en nuevo país?

- ¿Qué te facilitó la adaptación?

Paro conocer un fragmento de la realidad de la migración, es cierto, que se empieza por conocer el entorno donde se incluyen los migrantes y es cierto que en un país que no es 
el propio las posesiones se llevan en una maleta como lo describe Anne Maître sobre la escultura del migrante de Bruno Catalano; en este sentido, con lo poco que materialmente poseen, con las herramientas psicosociales que han desarrollado durante su vida y las escasas redes sociales que tienen los adolescentes deben adentrarse a conocer la nueva vida y su dinámica: "Haber empezado de cero, sin conocer nada o sea todo es nuevo y uno tiene que acoplarse a todo y seguir adelante..." “...sin embargo, me facilitó el hacer amigos y solidarizarme con la familia y los" (siglas de informante o simplemente informante 1).

Mediante este diálogo, es posible que confirmar que la percepción que tienen los adolescentes sobre su proceso de asentamiento es que en este se debe contar con la familia ya que finalmente se harán redes de amigos porque los jóvenes tienen la capacidad de establecer nuevas relaciones entre iguales que les pueden favorecer.

De igual manera los informantes mencionan dificultades con expresiones: "El venirme de mi país" que sería reconocer el amor por su patria y dejarla como uno de esos dolores del alma; ahora bien llama la atención que según su criterio dicen que "Acá es más difícil porque el trabajo es más duro" es de precisar que en este caso se refieren al trabajo que realizan sus padres y que ellos perciben como de mayor esfuerzo físico-laboral que los realizados en Venezuela y que tiene que ver con la duración de las jornadas que cuando son de empleos irregulares o informales se suelen extender de una manera incierta.

El papel de la institución educativa entra en juego para fortalecer la formación afectiva y emocional del adolescente migrado, sin embargo, no logra sustituir la presencia activa de los padres en la formación humana. En relación con las dificultades relacionadas con el proceso de adaptación, existen varios ejemplos que muestran el vínculo con su país de origen, por ejemplo, cuando las personas tienen que partir o multiplicar sus esfuerzos en los dos países para alcanzar una economía estable con la que puedan ofrecer la seguridad de la educación de sus hijos: “...que mi mamá tiene que estar viajando a Venezuela y ella tiene un negocio y yo me quedo". Informante.

En cuanto a las circunstancias que facilitan el proceso de adaptación social, se recuperaron narraciones relacionadas con el asentamiento en el territorio colombiano, por ejemplo, el hecho de que ya se tenía un conocimiento previo no solo del lugar sino también de la idiosincrasia del pueblo colombiano: "Que ya había venido antes con mi mamá en vacaciones y ya conocía algo y mi abuela vive aquí o sea que tenemos familia". Informante.

Ya se ha dicho que el presente estudio tiene un carácter de aproximación y por tanto se establece redes de relaciones que van entretejiéndose porque una categoría va relacionada con la otra y aquí aparece uno de los puntos tal vez más relevantes porque la apertura en los escenarios educativos es una de las alternativas más grandes que ayudan a tener una visión distinta en las crisis migratorias. Uno de los adolescentes expresa: “...lo fácil que me gusta los estudios de acá que la gente me ha tratado bien".

A continuación, se describen dos procesos identificados en relación con las experiencias de adaptación al contexto colombiano. Estos elementos identificados se convierten categorías de análisis que facilitan la comprensión e interpretación del fenómeno. 


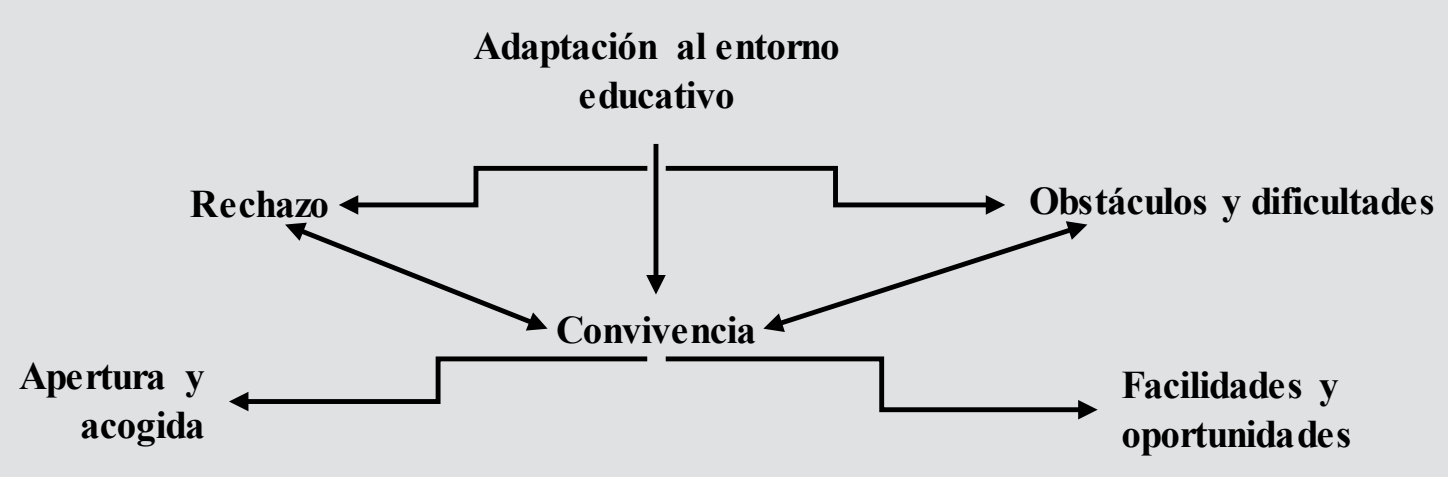

Figura 1. Adaptación al entorno educativo desde la perspectiva de los estudiantes

\section{a) Adaptación al Entorno Educativo}

Después de conocer de primera mano las experiencias al entrar en nuevo país es importante destacar el proceso de entrada al entorno educativo, para ello se seleccionó la pregunta de la guía de investigación ¿Cómo describirías las primeras semanas en el colegio: que cosas nuevas has visto o sentido, que experiencia nos cuentas?: "Pues un poco triste ya que deje unos compañeros allá en Venezuela que todavía están allá y pues las dos primeras semanas no hable con nadie por pena, miedo..." Informante.

En el cual se reconocen sensaciones afectivas presentes en su proceso de adaptación relacionadas con emociones negativas en las que se reconoce el dolor, la tristeza y cierto sentimiento de vacío tras la migración o síndrome de Ulises como lo expresan Reig, Clemente \& Sangiao (2018). Sin embargo, también se presentan contrastes relacionados con la apertura a las nuevas experiencias que la institución educativa ofrece, que serían las integraciones por medio de jornadas pedagógicas de aprendizaje de tipo lúdico, involucrando diferentes dimensiones de la formación:

a. "Las primeras semanas fueron muy interesantes ya que había actividades que yo nunca había realizado pues allá no se hacían". Informante.

b. "Pues a mí me gusto pues es un ambiente fresco muy agradable y mis compañeros me recibieron muy bien, creo que he creado un vínculo muy importante con mis compañeros". Informante.

c. "Las primeras semanas fue agradable, bastante bien. Fue bastante agradable porque uno va conociendo nuevas cosas por ejemplo el sistema de evaluaciones es diferente al de allá" Informante.

\section{b) Convivencia: apertura y acogida}

Si bien es cierto que todos los años entran a la institución nuevos estudiantes provenientes de la zona, es realmente novedoso recibir estudiantes que van llegando del vecino país y nunca se sabe cómo será el recibimiento y el ambiente escolar que se pueda desarrollar. A continuación, una estudiante expone la manera como sintió que lo recibieron: "Muy bien me han recibido, excelentemente, los quiero mucho porque ellos han sido muy buenos 
conmigo algo que yo no pensé que me iban a recibir de esa manera yo pensé que iba a ser algo diferente como la exclusión que porque yo soy venezolana entonces no y fue todo lo contrario". Informante.

En ese mismo sentido frases como "mis compañeros me recibieron muy bien, creo que he creado un vincula muy importante con mis compañeros" y "mis compañeros me empezaban hablar y cada vez más me sentía en confianza" develarían la función de la institución educativa como un ambiente propicio para la inclusión del estudiante en la comunidad, además de que la labor está repartida entre los actores del proceso educativo; por ejemplo, el siguiente testimonio deja claro que si en una comunidad educativa -estudiantes como docentes- aceptan positivamente al alumno migrado, el proceso de es un proceso rápido que solventará la experiencia emocional negativa: "la primera clase que tuve fue en el salón de informática y el profesor me pregunta si soy nuevo y se ofrece para darme apoyo en todo como todos los profesores, literal todos los profesores que me presentaron y que me presente me acogieron muy bien y para que, a los dos, tres días yo ya compaginaba con varios compañeros". Informante.

Sin embargo, existen expresiones que encienden algunas alarmas y ponen de manifiesto la necesidad de continuar sin descanso en la formación en temas de inclusión e integración con estudiantes y personas que viven el fenómeno de la migración, por ejemplo una estudiante decía "A mí me han tratado bien aunque a veces me siento como excluida por algunos como que no me toman en cuenta" esta es una realidad que no es ajena y que no es extraño que se dé y que se repita pero tampoco es extraño que se supere con facilidad y rapidez, lo que habría de fondo es la necesidad de una formación especial tanto para la ciudadanía, como para todas las Instituciones Educativas en la que se ofrezca luces que lleven a la aceptación e inclusión del otro o sea del migrante porque es una realidad nueva que sorprende y pone al descubierto las debilidades humanas de toda una sociedad y su cultura.

\section{CONCLUSIONES}

El fenómeno migratorio en la frontera colombo venezolana es una realidad que puede ser considera como una catástrofe humanitaria que con el paso del tiempo se agudiza y la cual requiere de atención desde el sistema, con la intención de prevenir o en su defecto dar atención a la salud mental de las personas en situación migratoria. Colombia ha recibido durante los últimos años de manera paulatina un número considerable de jóvenes que migran desde Venezuela y que ingresan a las Instituciones Educativas con el ánimo de recomponer su proyecto de vida.

Los jóvenes venezolanos que han ingresado a desarrollar sus procesos de formación escolar han sentido el cambio de cultura que les ha hecho vivir su propia crisis personal -muy común dentro de los procesos migratorios-, pero con el paso del tiempo se han venido modificando hasta lograr adaptarse positivamente a su nueva realidad. De acuerdo con la información recogida a través de las entrevistas con los alumnos migrantes que participaron, la acogida por parte de la comunidad educativa se muestra como agradable a través de una convivencia que les ofreció un clima escolar favorable para la inclusión social y la integración académica. 
Es importante destacar que para comprender la migración venezolana a Colombia habrá que considerar a la familia como unidad de análisis ya que, como afirman Torre \& Anguiano (2016), la orientación hacia el estudio del concepto de familia en los procesos migratorios responde a un comportamiento más proclive al colectivo que al actuar individual, de tal forma que el individuo prioriza a su familia sobre sí mismo, y supone una diferencia en cómo son y cómo actúan las familias respecto a otros grupos poblacionales. En este sentido la familia se convierte en promotor del desarrollo personal al impulsar al adolescente migrante a continuar con su preparación académica en el territorio de asentamiento.

Alarcon, Foulks, Vakkur (1998) retoman la noción de salud mental para analizar los procesos migratorios afirmando que las características individuales, además de la personalidad, también se encuentra presentes en el proceso de migrar. Ferrer, Palacio, Hoyos \& Madariaga (2014), evidencian los cambios que se experimentan individualmente relacionados con la migración, entre ellos son importantes destacar: la separación de la familia, la pérdida de amigos y grupos de iguales, el proceso de aculturación, el aprendizaje de una nueva lengua o hábitos culturales, además de experiencias emocionales traumáticas como la insistencia en el retorno, la consideración de del fracaso del proyecto migratorio, la incertidumbres por la condición de ilegalidad, y sentimientos de nostalgia y desarraigo como pueden verse en las narrativas de los alumnos migrantes entrevistados.

\section{REFERENCIAS}

ACNUR. (2012). Un año de crisis. Tendencias globales 2011. http://www.acnur.es/PDF/

Alarcon, E. Foulks y M. Vakkur (1998). Trastornos de personalidad y cultura: interacciones clínicas y conceptuales. Wiley.

Creswell, J. W. (2005). Educational research: Planning, conducting, and evaluating quantitative and qualitative research. Pearson Education, Inc.

Duque, N. H., \& Lasso, P. (2016). Autopercepción de Saberes y Prácticas sobre Educación y Desplazamiento Forzado en Docentes de Cali, Colombia. Revista Colombiana de Psicología, 25(1), 155-173. https://doi.org/10.15446/rcp.v25n1.49971

Ferrer, R., Palacio, J., Hoyos, O., \& Madariaga, C. (2014). Acculturation process and Immigrant's Adaptation: Individual characteristics and Social Networks. Psicología desde el Caribe, 31(3), 557-576. https://doi.org/10.14482/psdc.31.3.4766

García, J. (2002). Razón ética ante las migraciones. Ética y negocios. http://cepaim.org/ wp-content/uploads/2017/10/Modulo-02-CEPAIM-Reedici\%C3\%B3n-2016.pdf

Hernández, L. (2009). Población desplazada busca nuevas formas de organizarse. Universidad del Valle. http://aupec.univalle.edu.co/informes/2009/abril/ desplazados.html

Kearney, M., \& Bernadete, B. (2002). Procesos migratorios y relaciones interétnicas. FAAEE. 
Lasso, P. (2013). Cuando se vive el desarraigo. Educación y desplazamiento forzado: una mirada desde el Distrito de Aguablanca, Cali, Colombia. Revista Científica Guillermo de Ockham, 11(2), p 35-51. https://doi.org/10.21500/22563202.608

López, L. (2008). Personas desplazadas y educación. ECCA.

López-Sala, L. O. (2015). Inmigración en tiempos de crisis: dinámicas de movilidad emergentes y nuevos impactos sociales. Migraciones (37), 9-27. https://doi. org/10.14422/mig.i37.y2015.001

Patton, M. Q. (2014). Qualitative research \& evaluation methods: Integrating theory and practice. Sage publications. http://dissertation.argosy.edu/chicago/spring08/r7035_ sp08thakkar.doc

Ramírez, I. E. (1994). El modelo etnográfico en la investigación educativa. Educación, 3(5), 43-57. https://dialnet.unirioja.es/descarga/ articulo/5056972.pdf

Reig, A., Clemente, M.; \& Sangiao, I. (2018). Migración y síndrome de Ulises: Ser nadie en tierra de nadie. Barataria. Revista Castellano-Manchega de Ciencias Sociales, (24), 27-43. https://doi.org/10.20932/barataria.v0i24.388

Torre, E., \& Anguiano, M. (2016) Viviendo en las sombras: estrategias de adaptación de familias inmigrantes mexicanas en Arizona, 2007-2015. Papeles de población, 22(88), 171-207. http://www.scielo.org.mx/scielo.php?pid=S1405$74252016000200171 \&$ script $=$ sci_arttext\&tlng=pt

Vega, H. A. (2013). Características que evidencian el impacto educativo y cultural, a causa del fenómeno del desplazamiento forzado en Cúcuta. Educación y Humanismo, 15(25), p. 73-93. http://revistas.unisimon.edu.co/index.php/ educacion/article/download/2194/2086 